\title{
RATES OF GROWTH IN A CLASS OF HOMOGENEOUS MULTIDIMENSIONAL MARKOV CHAINS
}

\author{
M. GONZÁLEZ,* \\ R. MARTÍNEZ*** AND \\ M. MOTA, ${ }^{*}$ Universidad de Extremadura
}

\begin{abstract}
We investigate the asymptotic behaviour of homogeneous multidimensional Markov chains whose states have nonnegative integer components. We obtain growth rates for these models in a situation similar to the near-critical case for branching processes, provided that they converge to infinity with positive probability. Finally, the general theoretical results are applied to a class of controlled multitype branching process in which random control is allowed.
\end{abstract}

Keywords: Homogeneous multidimensional Markov chain; rate of growth; multitype branching process

2000 Mathematics Subject Classification: Primary 60J10

Secondary $60 \mathrm{~J} 80$

\section{Introduction}

One of the problems that is approached in the scientific literature on branching processes is the study of the growth rate of certain biological (human, animal, cell, etc.) or physical (particle, cosmic ray, etc.) populations. In the simplest models, such as the Bienaymé-GaltonWatson process, only geometric growth is possible when extinction does not occur. To a degree, the classical nondecomposable multitype Galton-Watson process somewhat inherits this dual behaviour of the one-dimensional model. Nonetheless, in some homogeneous modifications of these processes nonexponential rates of growth are also possible, particularly in the case known as critical or near critical.

In the present work, we deal with the problem of determining the rate of growth in a class of processes more general than (homogeneous) branching processes, namely homogeneous multidimensional Markov chains in discrete time taking values in the space of vectors with nonnegative integer components. The aim is to investigate what conditions must be imposed on such models in order to obtain nongeometric rates of growth, provided that there exists a positive probability of convergence to infinity. A detailed study of the indefinite growth of these chains was considered in [5], and conditions for their geometric growth can be found in [4].

We shall try to maintain the branching process and population dynamics perspective, and shall use their special terminology. An entire section of the paper will deal with controlled multitype branching processes with random control, a topic that has not previously been investigated.

Received 10 August 2005; revision received 23 December 2005.

* Postal address: Departamento de Matemáticas, Facultad de Ciencias, Universidad de Extremadura, 06071 Badajoz, Spain.

Supported by the Ministerio de Ciencia y Tecnología and the FEDER through the Plan Nacional de Investigación Científica, Desarrollo e Innovación Tecnológica, grant BFM2003-06074.

** Email address: rmartinez@unex.es 
Mathematically, we consider an $m$-dimensional homogeneous Markov chain, $\{Z(n)\}_{n \geq 0}$, whose states have nonnegative integer components (i.e. $S \subseteq \mathbb{N}_{0}^{m}$, where $S$ is the set of states). We refer to the chain as an HMMC. This chain can model the evolution of a population of $m$ different types of coexisting individual. More specifically, the $i$ th component of $Z(n)$ might represent the number of type- $i$ individuals $n$ generations after the process was started. The event 'explosion of the chain', denoted by $\mathscr{D}_{\infty}:=\{\|Z(n)\| \rightarrow \infty\}$, with $\|\cdot\|$ an arbitrary norm on $\mathbb{R}^{m}$, will play a fundamental role in our study and must be assumed to have positive probability.

In Section 2 we investigate the limiting behaviour of some sequences of linear functionals associated with HMMCs. After providing conditions for the event $\mathcal{D}_{\infty}$ to have positive probability, we show that, under certain conditions, they can be normalized on the explosion set by a sequence of constants with the same order as $\left\{n^{\alpha}\right\}_{n \geq 0}$, for some $\alpha>0$. In Section 3 we come back to the $m$-dimensional process $\{Z(n)\}_{n \geq 0}$ and prove that it is possible to find the same growth rate for such a process, again on the explosion set. Finally, in Section 4, we apply the results of Sections 2 and 3 to a class of controlled multitype branching process.

As indicated in the previous paragraph, for each $\mu \in \mathbb{R}_{+}^{m}$ we will consider the sequence of linear functionals $\{Z(n) \mu\}_{n \geq 0}$ associated with the chain $\{Z(n)\}_{n \geq 0}$. (Throughout, where no operator appears between vectors, scalar product is assumed.) This process is not a Markov chain, but it has some remarkable properties. Indeed $\mathscr{D}_{\infty}=\{Z(n) \mu \rightarrow \infty\}$, meaning that the explosion of the chain is equivalent to the unlimited growth of the sequence of functionals. In relation to this sequence of linear functionals we can introduce the variables $\xi_{n}^{\mu}, n \geq 0$, and the functions $g_{\mu}(z)$ and $\sigma_{\mu}^{2}(z)$, defined for every nonnull vector $z \in \mathbb{N}_{0}^{m}$ by

$$
\begin{aligned}
\xi_{n+1}^{\mu} & :=Z(n+1) \mu-\mathrm{E}[Z(n+1) \mu \mid Z(n)], \\
g_{\mu}(z) & :=\mathrm{E}[Z(n+1) \mu \mid Z(n)=z]-z \mu, \\
\sigma_{\mu}^{2}(z) & :=\operatorname{var}[Z(n+1) \mu \mid Z(n)=z] .
\end{aligned}
$$

Notice that they depend on the choice of the vector $\mu$, although in the rest of the paper, whenever there is no chance of ambiguity, we shall drop the use of $\mu$ in the notation and instead write $\xi_{n}$, $g(z)$, and $\sigma^{2}(z)$, respectively.

In order to determine nongeometric growth, we will consider vectors $\mu \in \mathbb{R}_{+}^{m}$ such that

$$
\lim _{\|z\| \rightarrow \infty} \frac{g(z)}{z \mu}=0
$$

which can be interpreted as meaning that the mean growth rate of the process $\{Z(n) \mu\}_{n \geq 0}$, i.e. $(z \mu)^{-1} \mathrm{E}[Z(n+1) \mu \mid Z(n)=z]$, is close to unity for sufficiently large $\|z\|$. Notice that (1.2) is an assumption on the Markov chain $\{Z(n)\}_{n \geq 0}$. This situation corresponds to the critical or near-critical case in branching processes.

\section{Asymptotic behaviour of $\{Z(n) \mu\}_{n \geq 0}$}

In this section, we search for sequences of constants with nongeometric growth that suitably normalize the sequence of linear functionals $\{Z(n) \mu\}_{n \geq 0}, \mu \in \mathbb{R}_{+}^{m}$, on the set $\mathcal{D}_{\infty}$. First we provide conditions, such as can be found in [5], which guarantee that $\mathrm{P}\left[\mathscr{D}_{\infty}\right]>0$.

Theorem 2.1. Let $z^{(0)} \in S$ be a vector such that

$$
\text { for every } C>0 \text { there exists an } n \geq 1 \text { such that } \mathrm{P}\left[Z(n) e>C \mid Z(0)=z^{(0)}\right]>0,
$$


with e being the m-dimensional vector whose components all equal unity. Assume that there exists a vector, $\mu \in \mathbb{R}_{+}^{m}$, for which

$$
\lim _{\|z\| \rightarrow \infty} \frac{g(z)}{z \mu}=0 \text { and } \liminf _{\|z\| \rightarrow \infty} \frac{2(z \mu) g(z)}{\sigma^{2}(z)}>1 .
$$

Suppose further that, for some $\delta, 0<\delta \leq 1$, and $\gamma>0$, the following equality holds:

$$
\mathrm{E}\left[\left|\xi_{n+1}\right|^{2+\delta} \mid Z(n)=z\right]=o\left(\frac{(z \mu)^{\delta} \sigma^{2}(z)}{\log (z \mu)^{1+\gamma}}\right) .
$$

Then $\mathrm{P}\left[\|Z(n)\| \rightarrow \infty \mid Z(0)=z^{(0)}\right]>0$.

Remark 2.1. Under condition (1.2), in [5] it was found that $\mathrm{P}\left[\mathcal{D}_{\infty}\right]=0$ if

$$
\limsup _{\|z\| \rightarrow \infty} \frac{2(z \mu) g(z)}{\sigma^{2}(z)}<1
$$

and, for some $\delta, 0<\delta<1$,

$$
\mathrm{E}\left[\left|\xi_{n+1}\right|^{2+\delta} \mid Z(n)=z\right]=o\left((z \mu)^{1+\delta} g(z)\right) .
$$

Remark 2.2. For the classical multitype branching process with irreducible matrix of means, $(z \mu)^{-1} g(z)=\rho-1$ for every nonnull $z \in \mathbb{N}_{0}^{m}$, with $\rho$ being the Perron-Frobenius eigenvalue associated to the matrix of means and $\mu \in \mathbb{R}_{+}^{m}$ a right eigenvector associated to $\rho$ (see [14, pp. 3-4]). In this case, $\mathrm{P}\left[\mathscr{D}_{\infty}\right]>0$ if and only if $\rho>1$. Moreover, $\{Z(n) \mu\}_{n \geq 0}$ presents geometric growth on $\mathscr{D}_{\infty}$ with rate $\rho$ (see [13, p. 20]). Notice that, for this process, condition (1.2) (critical case) implies that $\mathrm{P}\left[\mathscr{D}_{\infty}\right]=0$.

Let us now consider the sequence $\{Z(n) \mu\}_{n \geq 0}, \mu \in \mathbb{R}_{+}^{m}$. Suppose that there exist positive real functions, $\bar{g}(x)$ and $\bar{\sigma}^{2}(x)$, such that $g(z)=\bar{g}(z \mu)$ and $\sigma^{2}(z)=\bar{\sigma}^{2}(z \mu)$ for every vector $z \in \mathbb{N}_{0}^{m}$, satisfying the following assumptions.

(A1) $\bar{g}(x)=c x^{\alpha}+o\left(x^{\alpha}\right)$ for all $x>0$ and some $\alpha<1$ and $c>0$.

(A2) $\bar{\sigma}^{2}(x)=v x^{\beta}+o\left(x^{\beta}\right)$ for all $x>0$ and some $\beta \leq 1+\alpha$ and $v>0$.

(A3) $\mathrm{E}\left[\left|\xi_{n+1}\right|^{2+\delta} \mid Z(n)=z\right]=O\left(\sigma^{2+\delta}(z)\right)$ for some $\delta, 0<\delta \leq 1$.

Since $\alpha<1$, assumption (A1) implies that condition (1.2) holds. For mathematical reasons, we consider $\bar{g}(x)$ to be twice continuously differentiable and $\bar{\sigma}^{2}(x)$ to be continuously differentiable.

Remark 2.3. If (A1)-(A3) are satisfied and $z^{(0)} \in S$ is a vector such that (2.1) holds, then $\mathscr{D}_{\infty}$ has positive probability. Indeed, since

$$
\liminf _{\|z\| \rightarrow \infty} \frac{2(z \mu) g(z)}{\sigma^{2}(z)}=\liminf _{\|z\| \rightarrow \infty} \frac{2 c(z \mu)^{1+\alpha-\beta}(1+o(1))}{v+o(1)}= \begin{cases}2 c / v & \text { if } \beta=1+\alpha, \\ \infty & \text { if } \beta<1+\alpha\end{cases}
$$

and

$$
\frac{\mathrm{E}\left[\left|\xi_{n+1}\right|^{2+\delta} \mid Z(n)=z\right] \log (z \mu)^{1+\gamma}}{(z \mu)^{\delta} \sigma^{2}(z)}=O\left((z \mu)^{(\beta-2) \delta / 2} \log (z \mu)^{1+\gamma}\right)
$$

for some constants $\delta, \gamma>0$, from Theorem 2.1 we deduce that $\mathrm{P}\left[\mathcal{D}_{\infty} \mid Z(0)=z^{(0)}\right]>0$ if either $\beta<1+\alpha$ or $\beta=1+\alpha$ and $v<2 c$. 
The next result summarizes the asymptotic behaviour of the process $\{Z(n) \mu\}_{n \geq 0}$ under conditions (A1)-(A3), assuming that $\mathscr{D}_{\infty}$ has positive probability. We denote by $\left\{a_{n}\right\}_{n \geq 0}$ the solution to the difference equation

$$
a_{0}=1, \quad a_{n+1}=a_{n}+\bar{g}\left(a_{n}\right), \quad n \geq 0 .
$$

It is a matter of straightforward computation to verify that the sequence $\left\{a_{n}\right\}_{n \geq 0}$ is asymptotically equivalent to $((1-\alpha) c n)^{1 /(1-\alpha)}$.

Theorem 2.2. Assume (A1)-(A3) to hold, and that $\mathrm{P}\left[\mathcal{D}_{\infty}\right]>0$.

(a) If $\beta=1+\alpha$ and $v<2 c$ then, for all $x \in \mathbb{R}$,

$$
\lim _{n \rightarrow \infty} \mathrm{P}\left[\frac{(Z(n) \mu)^{1-\alpha}}{n} \leq x \mid \mathscr{D}_{\infty}\right]=\Gamma_{a, b}(x),
$$

where $\Gamma_{a, b}(x)$ denotes the gamma distribution function with parameters

$$
a:=(v(1-\alpha))^{-1}(2 c-v \alpha) \text { and } b:=2^{-1} v(1-\alpha)^{2} .
$$

(b) If $0<\alpha<1$ and $\beta<\alpha+1$ then,

(i) for $\beta<3 \alpha-1$, on $D_{\infty}, a_{n}^{-1} Z(n) \mu$ converges almost surely and in $L^{1}$ to 1 and $\bar{g}\left(a_{n}\right)^{-1}\left(Z(n) \mu-a_{n}\right)$ converges almost surely; and,

(ii) for $\beta \geq 3 \alpha-1$, on $\mathcal{D}_{\infty}, a_{n}^{-1} Z(n) \mu$ converges to 1 in $L^{1}$ and, for all $x \in \mathbb{R}$,

$$
\lim _{n \rightarrow \infty} \mathrm{P}\left[\Delta_{n}^{-1 / 2} \frac{Z(n) \mu-a_{n}}{\bar{g}\left(a_{n}\right)} \leq x \mid \mathscr{D}_{\infty}\right]=\Phi(x),
$$

with $\Phi(x)$ being the standard normal distribution function and

$$
\Delta_{n}:= \begin{cases}\frac{v}{c^{3}(1-\alpha)} \log n & \text { if } \beta=3 \alpha-1, \\ \frac{v}{\beta-3 \alpha+1} c^{(\beta-2) /(1-\alpha)}((1-\alpha) n)^{(\beta-3 \alpha+1) /(1-\alpha)} & \text { if } \beta>3 \alpha-1 .\end{cases}
$$

Proof. With the notation introduced in (1.1), we decompose the process $\{Z(n) \mu\}_{n \geq 0}$ as the following stochastic difference equation:

$$
Z(n+1) \mu=Z(n) \mu+g(Z(n))+\xi_{n+1}=Z(n) \mu+\bar{g}(Z(n) \mu)+\xi_{n+1}, \quad n \geq 0 .
$$

Let us define the function $G(x):=\int_{1}^{x} \mathrm{~d} y / \bar{g}(y)$, and check that the assumptions of Theorem 1 of [11] are fulfilled; namely that

$$
\lim _{x \rightarrow \infty} \bar{g}^{\prime}(x) G(x)=\frac{1}{1-\alpha}-1 \quad \text { and } \quad \lim _{x \rightarrow \infty} \frac{\bar{\sigma}^{2}(x)}{\bar{g}^{2}(x) G(x)}=\frac{v(1-\alpha)}{c} .
$$

This is in fact immediate, given that both conditions (A1)-(A3), with $\beta=1+\alpha$, and the equivalence

$$
G(x) \sim(c(1-\alpha))^{-1} x^{1-\alpha},
$$


hold. Then, by a direct application of Theorem 1 of [11], we obtain

$$
\lim _{n \rightarrow \infty} \mathrm{P}\left[\frac{G(Z(n) \mu)}{n} \leq x \mid \mathscr{D}_{\infty}\right]=\Gamma_{a, b^{\prime}}(x),
$$

with $a=(v(1-\alpha))^{-1}(2 c-v \alpha)$ and $b^{\prime}:=(2 c)^{-1} v(1-\alpha)$. If we now use (2.3) again and apply Slutsky's theorem, the proof of (a) is complete.

Here we must introduce some additional notation needed for the proof of (b). Let us rewrite (2.2) as

$$
Z(n+1) \mu=Z(n) \mu+\bar{g}(Z(n) \mu)\left(1+\eta_{n+1}\right), \quad n \geq 0,
$$

where $\eta_{n+1}:=\xi_{n+1} / \bar{g}(Z(n) \mu)$, at least on $\{g(Z(n)) \neq 0\}$. Upon defining the function $\bar{\varphi}^{2}(z):=\bar{g}^{-2}(x) \bar{\sigma}^{2}(x)$, it is immediate that $\bar{\varphi}^{2}(x) \sim v c^{-2} x^{\beta-2 \alpha}$. Also

$$
\mathrm{E}\left[\eta_{n+1} \mid Z(n)=z\right]=0, \quad \mathrm{E}\left[\eta_{n+1}^{2} \mid Z(n)=z\right]=\bar{\varphi}^{2}(z \mu),
$$

and, from (A3),

$$
\mathrm{E}\left[\left|\eta_{n+1}\right|^{2+\delta} \mid Z(n)=z\right]=O\left(\bar{\varphi}^{2+\delta}(z \mu)\right) .
$$

Upon further defining the function

$$
\psi(x):=\int_{1}^{x} \frac{\bar{\varphi}^{2}(y)}{\bar{g}(y)} \mathrm{d} y,
$$

from (A1) and (A2) we can easily derive the equivalence of $\beta \geq 3 \alpha-1$ and $\psi(\infty)=\infty$; more specifically,

$$
\psi(x) \sim \begin{cases}\frac{v}{c^{3}} \frac{1}{\beta-3 \alpha+1} x^{\beta-3 \alpha+1} & \text { if } \beta>3 \alpha-1, \\ \frac{v}{c^{3}} \log x & \text { if } \beta=3 \alpha-1 .\end{cases}
$$

Now, since (b) is a direct consequence of Theorem 3 of [10], we need only check the hypotheses of that theorem, as follows.

(A) From condition (1.2) we have $\bar{g}(x)=o(x)$. Moreover, since $0<\alpha<1, \bar{g}(x)$ is ultimately concave and $\bar{g}^{\prime}(x)$ ultimately convex.

(B) From $(2.3)$ we have $\tilde{G}(x) \sim(c(1-\alpha) x)^{1 /(1-\alpha)}, \tilde{G}$ being the inverse of $G$. Therefore, since $\beta<1+\alpha,\left(\bar{\varphi}^{2} \circ \tilde{G}\right)(x)$ is ultimately concave and

$$
\lim _{t \rightarrow \infty} \int_{1}^{t} x^{-2}\left(\bar{\varphi}^{2} \circ \tilde{G}\right)(x) \mathrm{d} x=\lim _{t \rightarrow \infty} \frac{v}{c^{2}}(c(1-\alpha))^{(\beta-2 \alpha) /(1-\alpha)} \int_{1}^{t} x^{(\beta-2) /(1-\alpha)} \mathrm{d} x<\infty .
$$

(C) The function $\left|\bar{g}^{\prime \prime}(x) \bar{g}(x) \bar{\varphi}^{-2}(x)\right|$ is equivalent to a positive multiple of $x^{4 \alpha-\beta-2}$; thus, it is ultimately decreasing if $\beta \geq 3 \alpha-1$ or, equivalently, $\psi(\infty)=\infty$. Also, if $\psi(\infty)<\infty$ then $\left|\bar{g}^{\prime \prime}(x) \bar{g}(x)\right|$ is equivalent to a positive multiple of $x^{2(\alpha-1)}$ and, since $\alpha<1$, is ultimately decreasing.

(D) Taking into account (2.4) and the fact that $\bar{g}^{\prime}(x) \sim c \alpha x^{\alpha-1}$, we have

$$
\bar{g}^{\prime}(x) \psi^{1 / 2}(x)=o(1) .
$$




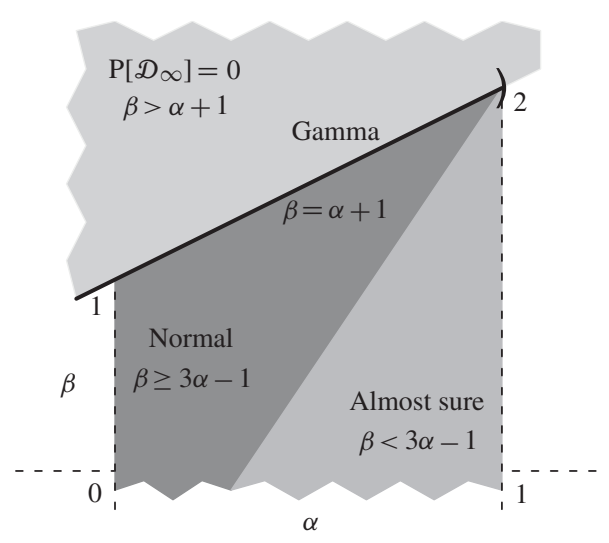

FIGURE 1.

Let $\hat{\psi}(x):=\psi \circ \tilde{G}(x)$. On the one hand, if $\beta<3 \alpha-1$ then $\hat{\psi}(\infty)<\infty$, and we obtain (b)(i) by applying Theorem 3(a) of [10], since $\{Z(n) \mu\}_{n \geq 0}$ is a sequence of nonnegative random variables. On the other hand, if $\beta \geq 3 \alpha-1$ then

$$
\hat{\psi}(x) \sim \begin{cases}\frac{v}{\beta-3 \alpha+1} c^{(\beta-2) /(1-\alpha)}((1-\alpha) x)^{(\beta-3 \alpha+1) /(1-\alpha)} & \text { if } \beta>3 \alpha-1, \\ \frac{v}{c^{3}(1-\alpha)} \log x & \text { if } \beta=3 \alpha-1,\end{cases}
$$

and, consequently, $\hat{\psi}(\infty)=\infty$. Then, by applying Theorem $3($ b) of [10] we obtain

$$
\lim _{n \rightarrow \infty} \mathrm{P}\left[\hat{\psi}(n)^{-1 / 2} \frac{Z(n) \mu-a_{n}}{\bar{g}\left(a_{n}\right)} \leq x \mid \mathcal{D}_{\infty}\right]=\Phi(x) .
$$

By using (2.5) again and applying Slutsky's theorem, (b)(ii) follows.

Remark 2.4. For different values of $\alpha$ and $\beta$, Figure 1 shows the different kinds of limiting behaviour that Theorem 2.2 predicts for the process $\{Z(n) \mu\}_{n \geq 0}$, when it is suitably normalized. Notice that if either $\beta=1+\alpha$ and $2 c<v$ or $\beta>1+\alpha$, then $\mathrm{P}\left[D_{\infty}\right]=0$ (see Remark 2.1).

Remark 2.5. If $\mu \in \mathbb{R}_{+}^{m}$ satisfies assumptions (A1)-(A3) then so does any other vector, $\bar{\mu} \in \mathbb{R}_{+}^{m}$, proportional to $\mu$, and, consequently, Theorem 2.2 remains true for the sequence of linear functionals $\{Z(n) \bar{\mu}\}_{n \geq 0}$, with the parameters of the limit distributions replaced by those corresponding to the vector $\bar{\mu}$.

In order to establish the next result, which is very important from a practical point of view, we also require

$$
\mathrm{P}[Z(n) \rightarrow 0]+\mathrm{P}[\|Z(n)\| \rightarrow \infty]=1,
$$

with 0 being the null state, to hold. This behaviour, typical of some homogeneous branching processes, is known as the 'extinction-explosion' duality, i.e. almost surely either the population becomes extinct or the total number of individuals grows indefinitely. Using Markov chain theory (see [2, p. 3]), it is easy to verify that if the null state is absorbing and every nonnull state is transient, then the chain satisfies (2.6). We observe that the condition that

$$
\mathrm{P}[Z(1)=0 \mid Z(0)=z]>0
$$


for all $z \in \mathbb{N}_{0}^{m}$ is sufficient for every nonnull state to be transient, obviously under the consideration of 0 being absorbing.

Proposition 2.1. Assume that (A1)-(A3), $0<\mathrm{P}\left[D_{\infty}\right]<1$, and (2.6) hold.

(a) Under the conditions of Theorem 2.2(a), the following statements hold for all $x \in \mathbb{R}$, with $a=(v(1-\alpha))^{-1}(2 c-v \alpha)$ and $b=2^{-1} v(1-\alpha)^{2}$ :

$$
\begin{gathered}
\lim _{n \rightarrow \infty} \mathrm{P}\left[\frac{(Z(n) \mu)^{1-\alpha}}{n} \leq x\right]=\mathrm{P}[Z(n) \rightarrow 0] \mathbf{1}_{\mathbb{R}_{0}}(x)+\mathrm{P}\left[D_{\infty}\right] \Gamma_{a, b}(x), \\
\lim _{n \rightarrow \infty} \mathrm{P}\left[\frac{(Z(n) \mu)^{1-\alpha}}{n} \leq x \mid\|Z(n)\|>0\right]=\Gamma_{a, b}(x) .
\end{gathered}
$$

Here $\mathbf{1}_{A}(\cdot)$ denotes the indicator function of the set $A$.

(b) Under the conditions of Theorem 2.2(b)(ii), the following statements hold for all $x \in \mathbb{R}$, with $\chi_{1}^{2}(x)$ being the chi-squared distribution function with one degree of freedom:

$$
\begin{gathered}
\lim _{n \rightarrow \infty} \mathrm{P}\left[\Delta_{n}^{-1} \frac{\left(Z(n) \mu-a_{n}\right)^{2}}{\bar{g}^{2}\left(a_{n}\right)} \leq x\right]=\mathrm{P}\left[\mathscr{D}_{\infty}\right] \chi_{1}^{2}(x), \\
\lim _{n \rightarrow \infty} \mathrm{P}\left[\Delta_{n}^{-1 / 2} \frac{Z(n) \mu-a_{n}}{\bar{g}\left(a_{n}\right)} \leq x \mid\|Z(n)\|>0\right]=\Phi(x) .
\end{gathered}
$$

Proof. Since $0<\mathrm{P}\left[D_{\infty}\right]<1$ and (2.6) holds, we deduce that $\mathrm{P}[Z(n) \rightarrow 0]>0$, which implies that the null state is absorbing. Indeed, since

$$
\mathrm{P}[Z(n) \rightarrow 0]=\lim _{n \rightarrow \infty} \mathrm{P}\left[\bigcap_{k=n}^{\infty}\{Z(k)=0\}\right]
$$

and, for each $n \geq 1$,

$$
\mathrm{P}\left[\bigcap_{k=n}^{\infty}\{Z(k)=0\}\right]=\lim _{s \rightarrow \infty} \mathrm{P}\left[\bigcap_{k=n}^{n+s}\{Z(k)=0\}\right]=\mathrm{P}[Z(n)=0] \lim _{s \rightarrow \infty} p_{00}^{s},
$$

with $p_{00}:=\mathrm{P}[Z(1)=0 \mid Z(0)=0]$, we find that $p_{00}=1$ and, therefore, that 0 is an absorbing state. Having established this, let us now prove the result.

(a) For simplicity, define $Y(n):=n^{-1}(Z(n) \mu)^{1-\alpha}, n \geq 1$. Since, for all $x>0$,

$$
\lim _{n \rightarrow \infty} \mathrm{P}[Y(n) \leq x \mid Z(r) \rightarrow 0]=1,
$$

we deduce that

$$
\lim _{n \rightarrow \infty} \mathrm{P}[Y(n) \leq x]=\mathrm{P}[Z(r) \rightarrow 0]+\mathrm{P}\left[\mathcal{D}_{\infty}\right] \lim _{n \rightarrow \infty} \mathrm{P}\left[Y(n) \leq x \mid \mathscr{D}_{\infty}\right],
$$

and, upon applying Theorem 2.2(a), obtain the proof of (a)(i). Now, since

$$
\begin{gathered}
\mathrm{P}\left[\mathscr{D}_{\infty}\right]=\lim _{n \rightarrow \infty} \mathrm{P}[\|Z(n)\|>0], \\
\lim _{n \rightarrow \infty} \mathrm{P}[\|Z(n)\|>0, Z(r) \rightarrow 0]=0, \\
\mathrm{P}\left[Y(n) \leq x,\|Z(n)\|>0, \mathscr{D}_{\infty}\right]=\mathrm{P}\left[Y(n) \leq x, D_{\infty}\right],
\end{gathered}
$$


we deduce that, for all $x \in \mathbb{R}$,

$$
\lim _{n \rightarrow \infty} \mathrm{P}[Y(n) \leq x \mid\|Z(n)\|>0]=\lim _{n \rightarrow \infty} \frac{\mathrm{P}\left[Y(n) \leq x \mid \mathscr{D}_{\infty}\right] \mathrm{P}\left[\mathscr{D}_{\infty}\right]}{\mathrm{P}[\|Z(n)\|>0]} .
$$

Upon again using Theorem 2.2(a), we obtain (a)(ii).

(b) In order to prove (b)(i), we define

$$
Y(n):=\Delta_{n}^{-1 / 2} \frac{Z(n) \mu-a_{n}}{\bar{g}\left(a_{n}\right)},
$$

and use a decomposition similar to (2.7) and the fact that $\Delta_{n}^{-1 / 2} \bar{g}^{-1}\left(a_{n}\right) a_{n}$ converges to $\infty$. Upon applying Theorem 2.2(b), the result follows. The proof of (b)(ii) follows the same steps, using

$$
Y(n):=\left(\Delta_{n}^{-1 / 2} \frac{Z(n) \mu-a_{n}}{\bar{g}\left(a_{n}\right)}\right)^{2} .
$$

Remark 2.6. Notice that the limit in (b)(i) is an improper distribution function. Moreover, from (b)(ii) we deduce that

$$
\lim _{n \rightarrow \infty} \mathrm{P}\left[\Delta_{n}^{-1} \frac{\left(Z(n) \mu-a_{n}\right)^{2}}{\bar{g}^{2}\left(a_{n}\right)} \leq x \mid\|Z(n)\|>0\right]=\chi_{1}^{2}(x),
$$

i.e. the chi-squared distribution function with one degree of freedom.

\section{Asymptotic behaviour of $\{Z(n)\}_{n \geq 0}$}

In the previous section, for every $\mu \in \mathbb{R}_{+}^{m}$ and under assumptions (A1)-(A3), we found sequences, $\left\{b_{n}\right\}_{n \geq 0}$, such that each $\left\{b_{n}^{-1} Z(n) \mu\right\}_{n \geq 0}$ converges to a nonnull random variable $W$, say, on $\mathscr{D}_{\infty}$, provided that this set has positive probability. As a consequence, we now prove the convergence of $\left\{b_{n}^{-1} Z(n)\right\}_{n \geq 0}$ to a random vector, $\tilde{W}$, that is concentrated in a one-dimensional subspace of $\mathbb{R}^{m}$ and whose magnitude is given by $W$.

We first need to introduce new notation. Let us impose the following condition on the transition vector of means of the chain:

$$
\mathrm{E}[Z(n+1) \mid Z(n)=z]=z \tilde{M}+\tilde{h}(z), \quad z \in \mathbb{N}_{0}^{m} .
$$

Here $\tilde{M}$ is a square matrix of order $m$ with nonnegative coefficients, and $\tilde{h}(z)$ is a function from $\mathbb{R}^{m}$ to $\mathbb{R}^{m}$ such that $\tilde{h}_{j}(z)=o(\|z\|)$ for all $j \in\{1, \ldots, m\}$. We also assume the matrix $\tilde{M}$ to be positively regular, meaning that if $\tilde{\rho}$ is its Perron-Frobenius eigenvalue and $\tilde{\mu} \in \mathbb{R}_{+}^{m}$ one of its associated right eigenvectors (see [14, pp. 3-4]), then $g_{\tilde{\mu}}(z)=(z \tilde{\mu})(\tilde{\rho}-1)+\tilde{h}(z) \tilde{\mu}$. Consequently (1.2) is equivalent to $\tilde{\rho}=1$.

Let $\tilde{\mu}^{(1)}, \ldots, \tilde{\mu}^{(m)}$ be a basis of right eigenvectors and right generalized eigenvectors of $\tilde{M}$ such that $\tilde{\mu}^{(1)}=\tilde{\mu}$ and $\tilde{v} \in \mathbb{R}_{+}^{m}$ is the left eigenvector associated to $\tilde{\rho}=1$, satisfying $\tilde{v} \tilde{\mu}=1$ and, consequently, $\tilde{v} \tilde{\mu}^{(i)}=0$ for each $i \in\{2, \ldots, m\}$. Finally, define $G^{(i)}(z)$, for each $z \in \mathbb{N}_{0}^{m}$ and $i \in\{1, \ldots, m\}$, by

$$
\begin{aligned}
G^{(i)}(z) & :=\mathrm{E}\left[\left|\xi_{n+1}^{\tilde{\mu}^{(i)}}\right| \mid Z(n)=z\right] \\
& =\mathrm{E}\left[\left|Z(n+1) \tilde{\mu}^{(i)}-\mathrm{E}\left[Z(n+1) \tilde{\mu}^{(i)} \mid Z(n)=z\right]\right| \mid Z(n)=z\right] .
\end{aligned}
$$

We can now formulate the following result. 
Theorem 3.1. Assume that (3.1) holds and that $\mathrm{E}[Z(0) \tilde{\mu}]<\infty, \mathrm{P}\left[\mathscr{D}_{\infty}\right]>0$, and (A1)-(A3) hold for the vector $\tilde{\mu}$. Suppose further that there exist constants $\delta_{1}, \delta_{2}<1$ such that

(i) $\left|\tilde{h}(z) \tilde{\mu}^{(i)}\right|=O\left((z \tilde{\mu})^{\delta_{1}}\right)$ for all $i \in\{2, \ldots, m\}$,

(ii) $G^{(i)}(z)=O\left((z \tilde{\mu})^{\delta_{2}}\right)$ for all $i \in\{2, \ldots, m\}$.

(a) If $\beta=1+\alpha$ and $v<2 c$ then, for every vector $\bar{x}=\left(\bar{x}_{1}, \ldots, \bar{x}_{m}\right) \in \mathbb{R}^{m}$,

$$
\lim _{n \rightarrow \infty} \mathrm{P}\left[\frac{Z(n)}{n^{1 /(1-\alpha)}} \leq \bar{x} \mid \mathscr{D}_{\infty}\right]=F_{\tilde{\nu} Z}(\bar{x}),
$$

with $F_{\tilde{v} Z}(\bar{x})$ being the distribution function associated with the random vector $\tilde{v} Z$, and $Z$ being a random variable such that $Z^{1-\alpha}$ follows a gamma distribution with parameters

$$
(v(1-\alpha))^{-1}(2 c-v \alpha) \text { and } 2^{-1} v(1-\alpha)^{2} .
$$

Vector inequalities are evaluated componentwise.

(b) If $0<\alpha<1$ and $\beta<\alpha+1$ then, on $\mathscr{D}_{\infty}, n^{-1 /(1-\alpha)} Z(n)$ converges to $(c(1-\alpha))^{1 /(1-\alpha)} \tilde{v}$ in $L^{1}$. Moreover, if $\beta \geq 3 \alpha-1$ and $2 \max \left\{\delta_{1}, \delta_{2}\right\}<(\beta-\alpha+1)$ then, for every vector $\bar{x} \in \mathbb{R}^{m}$, we have

$$
\lim _{n \rightarrow \infty} \mathrm{P}\left[\frac{Z(n)-\tilde{v} a_{n}}{\Lambda_{n}} \leq \bar{x} \mid \mathcal{D}_{\infty}\right]=F_{\tilde{v} U}(\bar{x}),
$$

with $F_{\tilde{v} U}(\bar{x})$ being the distribution function associated with the random vector $\tilde{v} U, U$ being a random variable with standard normal distribution, and $\Lambda_{n}$ satisfying

$$
\Lambda_{n}:= \begin{cases}v^{1 / 2}(c(1-\alpha))^{(3 \alpha-1) / 2(1-\alpha)} n^{\alpha /(1-\alpha)}(\log n)^{1 / 2} & \text { if } \beta=3 \alpha-1, \\ \left(v(\beta-3 \alpha+1)^{-1} c^{\beta /(1-\alpha)}((1-\alpha) n)^{(\beta-\alpha+1) /(1-\alpha)}\right)^{1 / 2} & \text { if } \beta>3 \alpha-1 .\end{cases}
$$

Proof. To prove the result, we apply reasoning similar to that used in [12] in the context of population-size-dependent multitype branching processes.

Since $\tilde{M}$ is positively regular, the eigenvalue $\tilde{\rho}=1$ has multiplicity one, and any other eigenvalue of $\tilde{M}$, say $r$, satisfies $|r|<\tilde{\rho}$. Suppose that $r$ is an eigenvalue with multiplicity $s \geq 1$ and right generalized eigenvectors $\tilde{\mu}^{\left(i_{1}\right)}, \ldots, \tilde{\mu}^{\left(i_{s}\right)}$, i.e.

$$
\tilde{M} \tilde{\mu}^{\left(i_{1}\right)}=r \tilde{\mu}^{\left(i_{1}\right)}, \quad \tilde{M} \tilde{\mu}^{\left(i_{j}\right)}=r \tilde{\mu}^{\left(i_{j}\right)}+\tilde{\mu}^{\left(i_{j-1}\right)} \quad \text { for } j \in\{2, \ldots, s\} .
$$

Let us prove by induction on $j$ that, for each $j \in\{1, \ldots, s\}$,

$$
\lim _{n \rightarrow \infty} \frac{Z(n) \tilde{\mu}^{\left(i_{j}\right)}}{n^{1 /(1-\alpha)}}=0 \quad \text { in } L^{1} .
$$

For $j=1$, using (3.1), it is almost surely true that

$$
\begin{aligned}
Z(n+1) \tilde{\mu}^{\left(i_{1}\right)} & =\mathrm{E}\left[Z(n+1) \tilde{\mu}^{\left(i_{1}\right)} \mid Z(n)\right]+Z(n+1) \tilde{\mu}^{\left(i_{1}\right)}-\mathrm{E}\left[Z(n+1) \tilde{\mu}^{\left(i_{1}\right)} \mid Z(n)\right] \\
& =r Z(n) \tilde{\mu}^{\left(i_{1}\right)}+\tilde{h}(Z(n)) \tilde{\mu}^{\left(i_{1}\right)}+Z(n+1) \tilde{\mu}^{\left(i_{1}\right)}-\mathrm{E}\left[Z(n+1) \tilde{\mu}^{\left(i_{1}\right)} \mid Z(n)\right]
\end{aligned}
$$

and, hence,

$$
\mathrm{E}\left[\left|Z(n+1) \tilde{\mu}^{\left(i_{1}\right)}\right|\right] \leq|r| \mathrm{E}\left[\left|Z(n) \tilde{\mu}^{\left(i_{1}\right)}\right|\right]+\mathrm{E}\left[\left|\tilde{h}(Z(n)) \tilde{\mu}^{\left(i_{1}\right)}\right|\right]+\mathrm{E}\left[\left|G^{\left(i_{1}\right)}(Z(n))\right|\right] .
$$


From (i) and (ii), and taking into account the fact that, on $\mathscr{D}_{\infty}, n^{-1 /(1-\alpha)} Z(n) \tilde{\mu}$ converges in distribution to a nonnegative random variable (see Theorem 2.2), we conclude that, for some constants $\delta_{1}, \delta_{2}<1$,

$$
\begin{aligned}
\mathrm{E}\left[\left|\tilde{h}(Z(n)) \tilde{\mu}^{\left(i_{1}\right)}\right|\right] & =O\left(\mathrm{E}\left[(Z(n) \tilde{\mu})^{\delta_{1}}\right]\right)=O\left(n^{\delta_{1} /(1-\alpha)}\right), \\
\mathrm{E}\left[G^{\left(i_{1}\right)}(Z(n))\right] & =O\left(\mathrm{E}\left[(Z(n) \tilde{\mu})^{\delta_{2}}\right]\right)=O\left(n^{\delta_{2} /(1-\alpha)}\right) .
\end{aligned}
$$

From (3.3) we then find that, for all $n \geq 0$,

$$
\mathrm{E}\left[\left|Z(n+1) \tilde{\mu}^{\left(i_{1}\right)}\right|\right] \leq|r| \mathrm{E}\left[\left|Z(n) \tilde{\mu}^{\left(i_{1}\right)}\right|\right]+O\left(n^{\max \left\{\delta_{1}, \delta_{2}\right\} /(1-\alpha)}\right) .
$$

By applying an iterative process, for all $n \geq 0$ we can verify that

$$
\mathrm{E}\left[\left|Z(n) \tilde{\mu}^{\left(i_{1}\right)}\right|\right] \leq \sum_{k=1}^{n}|r|^{n-k} O\left(k^{\max \left\{\delta_{1}, \delta_{2}\right\} /(1-\alpha)}\right)+|r|^{n+1} \mathrm{E}\left[\left|Z(0) \tilde{\mu}^{\left(i_{1}\right)}\right|\right] .
$$

Therefore, since $|r|<1$ and $\max \left\{\delta_{1}, \delta_{2}\right\}<1$, we recover (3.2) for $j=1$. Also, if $j \in$ $\{2, \ldots, s\}$, and assuming that (3.2) holds for $1, \ldots, j-1$, through a decomposition similar to (3.3) we obtain

$$
\begin{aligned}
\mathrm{E}\left[\left|Z(n+1) \tilde{\mu}^{\left(i_{j}\right)}\right|\right] \leq & |r| \mathrm{E}\left[\left|Z(n) \tilde{\mu}^{\left(i_{j}\right)}\right|\right]+\mathrm{E}\left[\left|Z(n) \tilde{\mu}^{\left(i_{j-1}\right)}\right|\right] \\
& +\mathrm{E}\left[\left|\tilde{h}(Z(n)) \tilde{\mu}^{\left(i_{j}\right)}\right|\right]+\mathrm{E}\left[G^{\left(i_{j}\right)}(Z(n))\right],
\end{aligned}
$$

and, by reasoning analogous to the $j=1$ case, we recover (3.2) for $j \in\{2, \ldots, s\}$.

In order to finish the proof, let us consider any vector $\eta \in \mathbb{R}^{m}$ and denote by $\eta_{1}, \ldots, \eta_{m} \in \mathbb{C}$ its components in the basis $\tilde{\mu}^{(1)}, \ldots, \tilde{\mu}^{(m)}$. Since $\tilde{v} \tilde{\mu}=1$ and $\tilde{v} \tilde{\mu}^{(i)}=0$ for all $i \in\{2, \ldots, m\}$, we have $\eta_{1}=\tilde{v} \eta$. Moreover,

$$
\frac{Z(n) \eta}{n^{1 /(1-\alpha)}}=\sum_{i=1}^{m} \eta_{i} \frac{Z(n) \tilde{\mu}^{(i)}}{n^{1 /(1-\alpha)}}
$$

If $\alpha<1$ and $\beta=1+\alpha$, from Theorem 2.2(a) we deduce that, for all $x \in \mathbb{R}$,

$$
\lim _{n \rightarrow \infty} \mathrm{P}\left[\frac{(Z(n) \tilde{\mu})^{1-\alpha}}{n} \leq x \mid \mathscr{D}_{\infty}\right]=\Gamma_{a, b}(x),
$$

with $a=(v(1-\alpha))^{-1}(2 c-v \alpha)$ and $b=2^{-1} v(1-\alpha)^{2}$. Hence, by applying (3.2), (3.4), and Slutsky's theorem, we find that, for all $x \in \mathbb{R}$,

$$
\lim _{n \rightarrow \infty} \mathrm{P}\left[\frac{Z(n) \eta}{n^{1 /(1-\alpha)}} \leq x \mid \mathscr{D}_{\infty}\right]=F_{Z}\left(\frac{x}{\tilde{v} \eta}\right)
$$

with $F_{Z}(x)$ being the distribution function of a random variable, $Z$, such that $Z^{1-\alpha}$ follows a gamma distribution with parameters $a$ and $b$. Taking the Cramèr-Wold device into account, we conclude the proof of (a).

If $0<\alpha<1$ and $\beta<1+\alpha$, we deduce from Theorem 2.2(b) that, conditioned on $\mathscr{D}_{\infty}$,

$$
\lim _{n \rightarrow \infty} \frac{Z(n) \tilde{\mu}}{a_{n}}=1 \text { in } L^{1} .
$$


Given that $a_{n} \sim(c(1-\alpha) n)^{1 /(1-\alpha)}$, and again applying (3.2) and (3.4), we find that, for any $\eta \in \mathbb{R}^{m}$, conditioned on $\mathscr{D}_{\infty}$,

$$
\lim _{n \rightarrow \infty} \frac{Z(n) \eta}{n^{1 /(1-\alpha)}}=(c(1-\alpha))^{1 /(1-\alpha)} \tilde{v} \eta \quad \text { in } L^{1} .
$$

Therefore, the first part of (b) is proved by choosing the vectors $\eta$ appropriately.

Moreover, if $\beta \geq 3 \alpha-1$ we deduce from Theorem 2.2(b)(ii) that, for all $x \in \mathbb{R}$,

$$
\lim _{n \rightarrow \infty} \mathrm{P}\left[\Delta_{n}^{-1 / 2} \frac{Z(n) \tilde{\mu}-a_{n}}{\bar{g}\left(a_{n}\right)} \leq x \mid \mathscr{D}_{\infty}\right]=\Phi(x) .
$$

Since $2 \max \left\{\delta_{1}, \delta_{2}\right\}<\beta-\alpha+1$, by an argument similar to that used to prove (3.2), for each $i \in\{2, \ldots, m\}$ we obtain

$$
\lim _{n \rightarrow \infty} \frac{Z(n) \tilde{\mu}^{(i)}}{n^{\beta-\alpha+1 / 2(1-\alpha)}}=0 \quad \text { in } L^{1} .
$$

By (3.4), for any $\eta \in \mathbb{R}^{m}$ we have

$$
\frac{\left(Z(n)-a_{n} \tilde{v}\right) \eta}{\Lambda_{n}}=\tilde{v} \eta\left(\frac{Z(n) \tilde{\mu}-a_{n}}{\Lambda_{n}}\right)+\sum_{i=2}^{m} \eta_{i} \frac{Z(n) \tilde{\mu}^{(i)}}{\Lambda_{n}} .
$$

Since $a_{n} \sim((1-\alpha) c n)^{1 /(1-\alpha)}$ and $\bar{g}(x) \sim c x^{\alpha}$, by using (3.5), (3.6), and (3.7) we deduce the second part of $(b)$ by once more applying the Cramèr-Wold device and Slutsky's theorem.

Remark 3.1. As we indicated above, notice that the limit vector obtained has a fixed direction, given by $\tilde{v}$, and a random magnitude, given by the limit of the sequence $\{Z(n) \tilde{\mu}\}_{n \geq 0}$, suitably normalized.

Remark 3.2. Taking Remark 2.5 into consideration, we deduce that, under the assumptions of Theorem 3.1, the behaviour of an HMMC does not depend on the choice of the right eigenvector $\tilde{\mu} \in \mathbb{R}_{+}^{m}$.

The following result is more precise than Theorem 3.1 from a practical point of view. For the proof, omitted because it is similar to that of Proposition 2.1, it is necessary to again assume the chain's dual behaviour, given by (2.6).

Corollary 3.1. If (2.6) holds and $0<\mathrm{P}\left[\mathscr{D}_{\infty}\right]<1$ then, under the hypotheses of Theorem 3.1(a), for every vector $\bar{x} \in \mathbb{R}^{m}$ we have

$$
\begin{gathered}
\lim _{n \rightarrow \infty} \mathrm{P}\left[\frac{Z(n)}{n^{1 /(1-\alpha)}} \leq \bar{x}\right]=\mathrm{P}[Z(n) \rightarrow 0] \mathbf{1}_{\mathbb{R}_{0}^{m}}(\bar{x})+\mathrm{P}\left[\mathscr{D}_{\infty}\right] F_{\tilde{v} Z}(\bar{x}), \\
\lim _{n \rightarrow \infty} \mathrm{P}\left[\frac{Z(n)}{n^{1 /(1-\alpha)}} \leq \bar{x} \mid\|Z(n)\|>0\right]=F_{\tilde{\nu} Z}(\bar{x}),
\end{gathered}
$$

with $F_{\tilde{v} Z}(\bar{x})$ being the distribution function of the random vector $\tilde{v} Z$, and $Z$ a random variable such that $Z^{1-\alpha}$ follows a gamma distribution with parameters $(v(1-\alpha))^{-1}(2 c-v \alpha)$ and $2^{-1} v(1-\alpha)^{2}$.

Remark 3.3. If $\tilde{v} \in \mathbb{R}_{+}^{m}$ and $Z$ is a random variable with distribution function $F_{Z}(x)$, then the distribution function of the random vector $\tilde{v} Z$ is given by

$$
F_{\tilde{v} Z}(\bar{x})=F_{Z}\left(\min _{1 \leq i \leq m} \frac{\bar{x}_{i}}{\tilde{v}_{i}}\right) \quad \text { for } \bar{x} \in \mathbb{R}^{m} .
$$




\section{On controlled multitype branching processes}

Unlike the one-dimensional version, the controlled multitype branching process has received little attention in the scientific literature. Historically, the possibility of controlling the number of potential progenitors in the population was proposed deterministically in [15] in both the univariate and the multidimensional cases. Random control was considered in [6], [7], [8], [9], and [17], for the univariate case only. In this section we shall apply the results obtained for the HMMCs to a new multitype branching model, called the controlled multitype branching process with random control, in which the number of progenitors of each type is controlled by means of a random mechanism. Furthermore, dependence between the individuals of the same generation at reproduction time is allowed. This is a major novel feature with respect to the classical branching models. Mathematically, we consider a sequence of $m$-dimensional random vectors, $\{Z(n)\}_{n \geq 0}$, defined recursively by

$$
Z(0)=z \in \mathbb{N}_{0}^{m}, \quad Z(n+1)=\sum_{i=1}^{m} \sum_{j=1}^{\phi_{i}^{n}(Z(n))} X^{i, n, j}, \quad n \geq 0 .
$$

Here $\left\{X^{i, n, j}, i=1, \ldots, m, n=0,1, \ldots, j=1,2, \ldots\right\}$ and $\left\{\phi^{n}(z), n=0,1, \ldots, z \in \mathbb{N}_{0}^{m}\right\}$ are two independent sequences of $m$-dimensional, nonnegative, integer-valued random vectors, defined on a common probability triple $(\Omega, \mathcal{A}, \mathrm{P})$, such that

(i) the stochastic processes $\left\{\phi^{n}(z), z \in \mathbb{N}_{0}^{m}\right\}, n=0,1, \ldots$, are independent and, for each $z \in \mathbb{N}_{0}^{m}$, the vectors $\left\{\phi^{n}(z), n=0,1, \ldots\right\}$ are identically distributed;

(ii) the stochastic processes $\left\{X^{i, n, j}, i=1, \ldots, m, j=1,2, \ldots\right\}, n=0,1, \ldots$, are independent and identically distributed, and, for each $i=1, \ldots, m$, the vectors

$$
\left\{X^{i, n, j}, n=0,1, \ldots, j=1,2, \ldots\right\}
$$

are identically distributed.

The sequence $\{Z(n)\}_{n \geq 0}$ is called a controlled multitype branching process with random control, abbreviated to CMP.

The controlled branching processes proposed in [15] and [17] can be deduced as particular cases of the CMP. Moreover, a CMP is an HMMC, and, taking into account the independence of control and reproduction, for every $z \in \mathbb{N}_{0}^{m}$ we have

$$
\mathrm{E}[Z(n+1) \mid Z(n)=z]=\mathrm{E}\left[\phi^{0}(z)\right] R
$$

with $R:=\left(r_{i j}\right)_{1 \leq i, j \leq m}$ being the square matrix of order $m$ with elements $r_{i j}:=\mathrm{E}\left[X_{j}^{i, 0,1}\right]$.

In the present study we assume that, for each type, $i=1, \ldots, m$, there exist $\phi_{i} \geq 0$ and $h_{i}(z)$ such that

$$
\mathrm{E}\left[\phi_{i}^{0}(z)\right]=z_{i} \phi_{i}+h_{i}(z) \quad \text { and } \quad h_{i}(z)=o(\|z\|) .
$$

Then, by (4.1), (3.1) holds with the matrix $\tilde{M}$ given by the elements $\tilde{m}_{i j}:=\phi_{i} r_{i j}, i, j=$ $1, \ldots, m$, and with $\tilde{h}_{j}(z):=\sum_{i=1}^{m} h_{i}(z) r_{i j}, j=1, \ldots, m$. This condition means that the average number of potential progenitors of each type in a generation is proportional to the number of individuals of this type plus or minus a certain quantity of individuals that is negligible compared to the total population size. Notice that, under assumption (4.2), immigration and emigration of progenitors of each type is allowed. Immigration is possible even if there are no 
individuals of a given type. This does not occur if $h_{i}(z)=z_{i} o(1)$. In this case, however, we can determine $\phi_{i}$ explicitly, as

$$
\phi_{i}=\lim _{\|z\| \rightarrow \infty, z_{i} \neq 0} \frac{\mathrm{E}\left[\phi_{i}^{0}(z)\right]}{z_{i}} .
$$

The matrix $\left(\phi_{i} r_{i j}\right)_{1 \leq i, j \leq m}$ is irreducible if and only if $\phi_{i}$ is nonnull for all $i=1, \ldots, m$ and the matrix $R$ is irreducible. In this case, if $\tilde{\rho}$ is the Perron-Frobenius eigenvalue of $\left(\phi_{i} r_{i j}\right)_{1 \leq i, j \leq m}$, (1.2) holds if and only if $\tilde{\rho}=1$, taking $\mu=\tilde{\mu} \in \mathbb{R}_{+}^{m}$ to be one of the right eigenvectors of this matrix associated to $\tilde{\rho}$.

In order to apply the results proved in the previous section, let us conveniently bound $\mathrm{E}\left[\left|\xi_{n+1}\right|^{2+\delta} \mid Z(n)=z\right]$ and $G^{(i)}(z)$ for $\delta>0, i=1, \ldots, m$, and $z \in \mathbb{N}_{0}^{m}$. We have

$$
\mathrm{E}\left[\left|\xi_{n+1}\right|^{2+\delta} \mid Z(n)=z\right]=\mathrm{E}\left[\left|\sum_{i=1}^{m}\left(\sum_{j=1}^{\phi_{i}^{n}(z)} X^{i, n, j} \tilde{\mu}-\mathrm{E}\left[\phi_{i}^{n}(z)\right] \mathrm{E}\left[X^{i, n, 1}\right] \tilde{\mu}\right)\right|^{2+\delta}\right] .
$$

Taking into account the fact that

$$
\begin{aligned}
\sum_{j=1}^{\phi_{i}^{n}(z)} X^{i, n, j} \tilde{\mu}-\mathrm{E}\left[\phi_{i}^{n}(z)\right] \mathrm{E}\left[X^{i, n, 1}\right] \tilde{\mu}= & \sum_{j=1}^{\phi_{i}^{n}(z)}\left(X^{i, n, j}-\mathrm{E}\left[X^{i, n, 1}\right]\right) \tilde{\mu} \\
& +\left(\phi_{i}^{n}(z)-\mathrm{E}\left[\phi_{i}^{n}(z)\right]\right) \mathrm{E}\left[X^{i, n, 1}\right] \tilde{\mu},
\end{aligned}
$$

from the $C_{r}$-inequality we obtain

$$
\begin{aligned}
\mathrm{E}\left[\left|\xi_{n+1}\right|^{2+\delta} \mid Z(n)=z\right] \leq & A_{1} \sum_{i=1}^{m} \mathrm{E}\left[\left|\sum_{j=1}^{\phi_{i}^{n}(z)}\left(X^{i, n, j}-\mathrm{E}\left[X^{i, n, 1}\right]\right) \tilde{\mu}\right|^{2+\delta}\right] \\
& +A_{2} \sum_{i=1}^{m} \mathrm{E}\left[\left|\phi_{i}^{n}(z)-\mathrm{E}\left[\phi_{i}^{n}(z)\right]\right|^{2+\delta}\right]\left(\mathrm{E}\left[X^{i, n, 1}\right] \tilde{\mu}\right)^{2+\delta}
\end{aligned}
$$

for certain constants $A_{1}, A_{2}>0$. Moreover, in the general case, i.e. when the random vectors $X^{i, n, j}, j=1,2, \ldots, i=1, \ldots, m$, are not necessarily independent for each fixed $n \geq 0$, it can be shown that

$$
\mathrm{E}\left[\left|\sum_{j=1}^{\phi_{i}^{n}(z)}\left(X^{i, n, j}-\mathrm{E}\left[X^{i, n, 1}\right]\right) \tilde{\mu}\right|^{2+\delta}\right] \leq \mathrm{E}\left[\phi_{i}^{n}(z)^{2+\delta}\right] \mathrm{E}\left[\left|\left(X^{i, n, 1}-\mathrm{E}\left[X^{i, n, 1}\right]\right) \tilde{\mu}\right|^{2+\delta}\right] .
$$

If, however, these vectors are independent, then the Marcinkiewicz-Zygmund inequality (see [1, p. 386]), can be used to bound (4.4) by

$$
\mathrm{E}\left[\phi_{i}^{n}(z)^{1+\delta / 2}\right] \mathrm{E}\left[\left|\left(X^{i, n, 1}-\mathrm{E}\left[X^{i, n, 1}\right]\right) \tilde{\mu}\right|^{2+\delta}\right] .
$$

Taking its definition into account, we bound $G^{(i)}(z)$ by

$$
G^{(i)}(z) \leq \sum_{i=1}^{m} \sum_{j=1}^{m}\left|\tilde{\mu}_{j}^{(i)}\right| \mathrm{E}\left[\left|\sum_{k=1}^{\phi_{i}^{n}(z)} X_{j}^{i, n, k}-\mathrm{E}\left[\phi_{i}^{n}(z)\right] r_{i j}\right|\right]
$$


Under the independence assumption, and proceeding in the same way as in (4.3), we can obtain the following bound from the von Bahr-Esseen inequality (see [16]), for some $\tilde{\alpha}, 1 \leq \tilde{\alpha} \leq 2$ :

$$
\begin{aligned}
\mathrm{E}\left[\left|\sum_{k=1}^{\phi_{i}^{n}(z)} X_{j}^{i, n, k}-\mathrm{E}\left[\phi_{i}^{n}(z)\right] r_{i j}\right|\right] \leq & \left(2 \mathrm{E}\left[\phi_{i}^{n}(z)\right] \mathrm{E}\left[\left|X_{j}^{i, n, 1}-r_{i j}\right|^{\tilde{\alpha}}\right]\right)^{1 / \tilde{\alpha}} \\
& +\left(\mathrm{E}\left[\left|\phi_{i}^{n}(z)-\mathrm{E}\left[\phi_{i}^{n}(z)\right]\right|^{\tilde{\alpha}}\right] \mathrm{E}\left[\left(X_{j}^{i, n, 1}\right)^{\tilde{\alpha}}\right]\right)^{1 / \tilde{\alpha}} .
\end{aligned}
$$

In order to guarantee that (2.1) holds, it is sufficient that, for every nonnull $z \in \mathbb{N}_{0}^{m}$,

$$
\mathrm{P}\left[\phi_{i}^{0}(z)>z_{i}, X^{i, 0, j} \neq 0, i \in I_{(z)}, j=1, \ldots, \phi_{i}^{0}(z)\right]>0,
$$

with $I_{(z)}=\left\{i \in\{1, \ldots, m\}: z_{i} \neq 0\right\}$. To summarize, we establish the following result for the CMP.

Corollary 4.1. Let $\{Z(n)\}_{n \geq 0}$ be a CMP satisfying (4.2), where $\left(\phi_{i} r_{i j}\right)_{1 \leq i, j \leq m}$ is a positively regular matrix with Perron-Frobenius eigenvalue $\tilde{\rho}=1$ and associated right eigenvector $\tilde{\mu} \in \mathbb{R}_{+}^{m}$. Suppose further that (4.5) holds and that, for every nonnull vector $z$,

(i) $h_{i}(z)=c_{i}(z \tilde{\mu})^{\alpha}+o\left((z \tilde{\mu})^{\alpha}\right)$ for each $i=1, \ldots$, m and for some $\alpha<1$ and $c_{i} \in \mathbb{R}$ such that $\sum_{i=1}^{m} \sum_{j=1}^{m} c_{i} r_{i j} \tilde{\mu}_{j}>0$,

(ii) $\bar{\sigma}^{2}(z \tilde{\mu}):=\operatorname{var}[Z(n+1) \tilde{\mu} \mid Z(n)=z]=v(z \tilde{\mu})^{\beta}+o\left((z \tilde{\mu})^{\beta}\right)$ for some $\beta \leq 1+\alpha$ and $v>0$,

(iii) $\max _{1 \leq i \leq m}\left\{\mathrm{E}\left[\left|\phi_{i}^{0}(z)-\mathrm{E}\left[\phi_{i}^{0}(z)\right]\right|^{2+\delta}\right], \mathrm{E}\left[\phi_{i}^{0}(z)^{2+\delta}\right]\right\}=O\left((\bar{\sigma}(z \tilde{\mu}))^{2+\delta}\right)$ for some $\delta, 0<$ $\delta \leq 1$,

(iv) $\max _{1 \leq i \leq m}\left|h_{i}(z)\right|=O\left((z \tilde{\mu})^{\delta_{1}}\right)$ for some $\delta_{1}<1$,

(v) $\max _{1 \leq i \leq m} \mathrm{E}\left[\left|\sum_{k=1}^{\phi_{i}^{n}(z)} X_{j}^{i, n, k}-\mathrm{E}\left[\phi_{i}^{n}(z)\right] r_{i j}\right|\right]=O\left((z \tilde{\mu})^{\delta_{2}}\right)$ for some $\delta_{2}<1$.

The statements of Theorem 3.1 then hold.

Remark 4.1. Notice that, under the independence assumption, parts (iii) and (v) of Corollary 4.1 can be respectively replaced by

$$
\max _{1 \leq i \leq m}\left\{\mathrm{E}\left[\left|\phi_{i}^{0}(z)-\mathrm{E}\left[\phi_{i}^{0}(z)\right]\right|^{2+\delta}\right], \mathrm{E}\left[\phi_{i}^{0}(z)^{1+\delta / 2}\right]\right\}=O\left((\bar{\sigma}(z \tilde{\mu}))^{2+\delta}\right)
$$

and, for some $\tilde{\alpha}, 1<\tilde{\alpha} \leq 2$,

$$
\max _{1 \leq i \leq m}\left\{\mathrm{E}\left[\phi_{i}^{0}(z)\right], \mathrm{E}\left[\left|\phi_{i}^{0}(z)-\mathrm{E}\left[\phi_{i}^{0}(z)\right]\right|^{\tilde{\alpha}}\right]\right\}=O\left((z \tilde{\mu})^{\tilde{\alpha} \delta_{2}}\right) .
$$

Remark 4.2. Under the conditions of Corollary 4.1 and the dual extinction-explosion behaviour, we can apply Proposition 2.1 and Corollary 3.1 to obtain results that are more precise from a practical point of view.

As we indicated before, in order to guarantee that (2.6) holds, it is sufficient to check both that the null state is absorbing and that every nonnull state is transient. For the CMP, it is easy to prove that 0 is absorbing if and only if $\mathrm{P}\left[\phi^{0}(0)=0\right]=1$, and that every nonnull vector $z \in \mathbb{N}_{0}^{m}$ is transient if

$$
\mathrm{P}\left[\bigcap_{i=1}^{m}\left(\left\{\phi_{i}^{0}(z)=0\right\} \cup\left\{\phi_{i}^{0}(z)>0, X^{i, 0, j}=0, j=1, \ldots, \phi_{i}^{0}(z)\right\}\right)\right]>0 .
$$



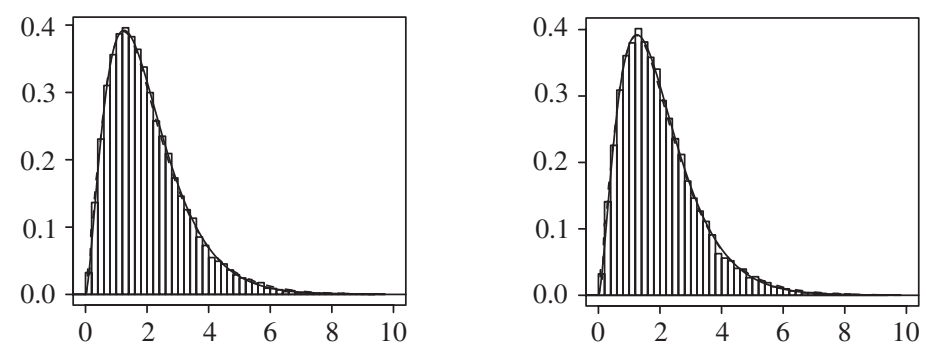

Figure 2.
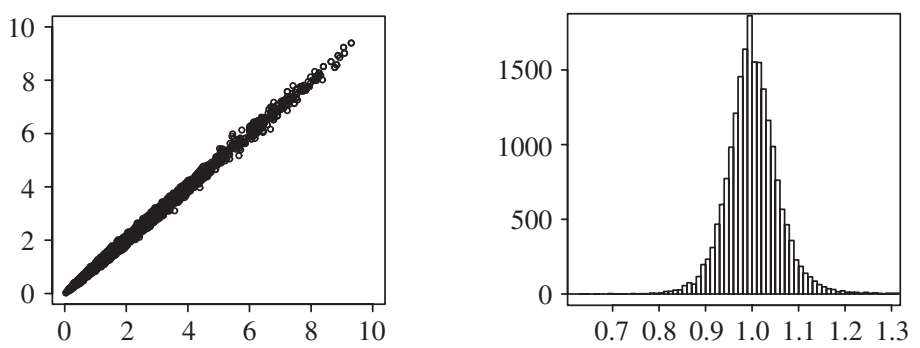

Figure 3.

By way of example, we consider a CMP with $m=2$, such that the random variables $\left\{X_{j}^{i, n, k}, i, j=1, \ldots, m, n=0,1, \ldots, k=1,2, \ldots\right\}$ are independent with mean and variance both equal to 1 , i.e. $r_{i j}=\operatorname{var}\left[X_{j}^{i, 0,1}\right]=1$. We also assume that the random variables

$$
\left\{\phi_{i}^{n}(z), i=1, \ldots, m, n=0,1, \ldots, z \in \mathbb{N}_{0}^{m}\right\}
$$

are independent and follow Poisson distributions, with $\mathrm{E}\left[\phi_{i}^{0}(z)\right]=0.5 z_{i}+1$.

It is not hard to prove that (3.1) holds, with $\tilde{\rho}=1, \tilde{\mu}=(1,1)$, and $\tilde{v}=(0.5,0.5)$. Furthermore, conditions (i), (ii), and (iv) of Corollary 4.1 are satisfied for $\alpha=0, \beta=1, c_{i}=1$, and $v=3$. Also, from the properties of the Poisson distribution we obtain conditions (4.6) and (4.7). Therefore, by applying Theorem 3.1(a) with $c=4$ we deduce that, for all $\bar{x} \in \mathbb{R}^{2}$,

$$
\lim _{n \rightarrow \infty} \mathrm{P}\left[\frac{Z(n)}{n} \leq \bar{x} \mid \mathscr{D}_{\infty}\right]=\Gamma_{8 / 3,3 / 2}\left(2 \min \left\{\bar{x}_{1}, \bar{x}_{2}\right\}\right)=\Gamma_{8 / 3,3 / 4}\left(\min \left\{\bar{x}_{1}, \bar{x}_{2}\right\}\right) .
$$

To illustrate this type of behaviour, we simulated a total of 20000 processes up to generation 500 of the above model, with $Z(0)=(1,2)$ and a reproduction law following an independent Poisson marginal distribution. Figure 2 shows the empirical distribution of $Z_{1}(500) / 500$ (lefthand plot) and $Z_{2}(500) / 500$ (right-hand plot), together with the density function of the limit variable $\Gamma_{8 / 3,3 / 4}$ (solid line) and a kernel density estimate for the positive distribution (dotted line).

Finally, we illustrate the behaviour of the vector $Z(500) / 500$. Figure 3 (left-hand plot) shows the sample space. The strong linear dependency given by the eigenvector $\tilde{v}=(0.5,0.5)$ can be clearly seen. This is related to the right-hand plot of Figure 3, which shows a histogram of $Z_{1}(500) / Z_{2}(500)$. Figure 4 shows a kernel density estimate of the joint density function of the vector $Z(500) / 500$. In it we observe the limiting behaviour of the process described above. 


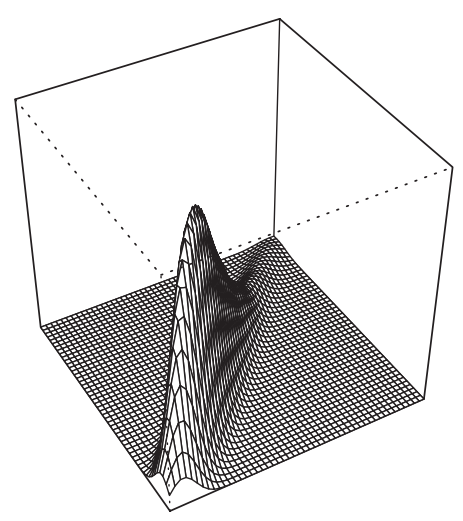

Figure 4.

Remark 4.3. For the computer simulation, we used the language and environment for statistical computing and graphics R ('GNU S') (see [3]).

\section{References}

[1] Chow, Y. S. And TeIcher, H. (1997). Probability Theory. Independence, Interchangeability, Martingales. Springer, New York.

[2] Chung, K. L. (1967). Markov Chains with Stationary Transition Probabilities, 2nd edn. Springer, New York.

[3] Gentleman, R. and Ihaka, R. (1996). R: a language for data analysis and graphics. J. Comput. Graph. Statist. 5, 299-314.

[4] González, M., Martínez, R. and Mota, M. (2005). On the geometric growth in a class of homogeneous multitype Markov chains. J. Appl. Prob. 42, 1015-1030.

[5] González, M., Martínez, R. and Mota, M. (2005). On the unlimited growth of a class of homogeneous multitype Markov chains. Bernoulli 11, 559-570.

[6] González, M., Molina, M. And Del Puerto, I. (2002). On the class of controlled branching processes with random control functions. J. Appl. Prob. 39, 804-815.

[7] González, M., Molina, M. And del Puerto, I. (2003). On the geometric growth in controlled branching processes with random control function. J. Appl. Prob. 40, 995-1006.

[8] González, M., Molina, M. and del Puerto, I. (2005). Asymptotic behaviour for the critical controlled branching process with random control function. J. Appl. Prob. 42, 463-477.

[9] González, M., Molina, M. ANd del Puerto, I. (2005). On $L^{2}$-convergence of controlled branching processes with random control function. Bernoulli 11, 37-46.

[10] Keller, G., Kersting, G. And Rösler, U. (1987). On the asymptotic behaviour of discrete time stochastic growth processes. Ann. Prob. 15, 305-343.

[11] Kersting, G. (1992). Asymptotic Г-distribution for stochastic difference equations. Stoch. Process. Appl. 40, 15-28.

[12] Klebaner, F. (1991). Asymptotic behaviour of near-critical multitype branching processes. J. Appl. Prob. 28, 512-519.

[13] Mode, C. J. (1971). Multitype Branching Processes. Elsevier, New York.

[14] Seneta, E. (1981). Nonnegative Matrices and Markov Chains, 2nd edn. Springer, New York.

[15] Sevast'yanov, B. A. and Zubkov, A. (1974). Controlled branching processes. Theory Prob. Appl. 19, 14-24.

[16] Von Bahr, B. And Esseen, C. G. (1965). Inequalities for the $r$ th absolute moment of a sum of random variables, $1 \leq r \leq 2$. Ann. Math. Statist. 36, 299-303.

[17] Yanev, N. (1975). Conditions for degeneracy of $\phi$-branching processes with random $\phi$. Theory Prob. Appl. 20, 421-428. 